\title{
Analysis of Different Modelling Techniques for Phase Ordering
}

\author{
J. Andrews ${ }^{1 *}$ and T. Sasikala ${ }^{2}$ \\ 'Research Scholar, Sathyabama University, Chennai, India; andrews_593@yahoo.com \\ 2Principal, SRR Engineering College, Padur, Chennai, India; sasi_madhu2k2@yahoo.co.in
}

\begin{abstract}
In this paper we have proposed Multi Variant Modelling Technique for selection and ordering of objective functions for compiler optimization. This model is evaluated in comparison with Linear and Interactive Modelling. Focus is mainly on Phase Order Searching. The result shows that performance achieved by multi variant modelling outperforms other modelling techniques using Mibench Benchmark applications.
\end{abstract}

Keywords: Compiler Optimization, Machine Learning, Benchmark, Modelling.

\section{Introduction}

Benchmark application requires a set of finetuned optimizations for achieving higher performance. Finding the best set of optimization requires deep understanding of system domain. Using iterative optimization, one can iteratively apply set of optimization sequences for an application. These sequences of optimization techniques are applied one by one until best set of sequence is obtained. However, finding the best set of sequences for a problem is a critical and extremely challenging task. Selection and ordering of optimization sequences for an application plays a major role for achieving higher optimization. Finding better ordering after many passes impacts higher performance as far as optimization is concerned. To find right optimization sequences efficiently, there is a need for searching the search space efficiently. For selecting the best set of sequences and ordering the sequences, multi variant models are developed using different machine learning strategy. The model is constructed using the characteristics of the code being optimized. Based on the characteristics of the program, the model can predict best set of sequences. Once the sequences are found, ordering of the selected sequences is done with which speed up can be measured. Applying optimization sequences based on these orderings improves optimization performance.

For testing various applications, Mibench Benchmark Application is used. These benchmark applications are compiled and executed using gcc or g++ compiler. Modern gcc compiler provides larger number of optimization techniques. Finding ordering would not be feasible by considering all possible combination of optimization sequences, i.e., exhaustive search of the orderings is not possible. Optimal set of ordering is specific to application only. No ordering is best for all kinds of applications. Optimal ordering based on the characteristics of the applications works well for some program, but not for all the programs. Addressing phase order search problem to select ordering various machine learning strategy is used.

Selection of ordering is based upon four important characteristics such as: 1) Program features extraction with respect to execution time 2) Various benchmark applications 3) Applying various machine learning strategy and 4) Classification.

Performing all the above steps requires choosing correct models. In this paper, we have proposed multi variant modelling. Multi variant modelling is able to

*Author for correspondence: 
predict optimization sequences effectively. The results are compared with other modelling techniques such as Linear Modelling and Iterative Modelling. To test the above models, a set of mibench benchmark applications ranging from simple applications to various scientific and embedded applications are used. Previously, the problem of finding optimal ordering addressed by Agakov et $\mathrm{al}^{3}$ did not explain various machine learning strategy. Andrews et al. ${ }^{11}$ gave an explanation for selecting set of program features. Triantafyllis et al. ${ }^{5}$ tackled the problem for reducing compile time and execution time. Cavazos et al. ${ }^{4}$ developed a model using the characteristics of the program and an optimization sequence. It predicts the output speed up related to default settings. Previously iterative compilation has been shown to outperform other techniques. Almagor et al. ${ }^{6}$ tried to reduce maximum number of evaluations. They devised a new algorithm that could outperform other techniques. Kulkarni et al., ${ }^{2,7}$ tried to reduce the running time by storing tested code in a database. Cooper et al. ${ }^{10}$ used genetic algorithm to find a solution for phase ordering problem. Several researchers tried machine learning strategy also. Stephenson et al. ${ }^{8}$ used genetic programming. Fursin et al. ${ }^{1}$ used static program features for performing optimization. Recent research shows that applications using dynamic program features outperform that of static program features. Cavazos et al. $^{4}$ uses supervised learning for creating models.

We evaluate different modelling techniques with various machine learning algorithms. Recent researchers proposed various optimization algorithms such as genetic algorithm; statistical techniques and random search are expensive. There is a need for automatically constructing prediction models which picks the best set of optimization sequence. The rest of the paper is organized as follows. Section 2 describes various modelling techniques. Section 3 describes various machine learning algorithms used for constructing models. Section 4 describes Results and discussions and Section 5 describes conclusions and future scope.

\section{Modelling Techniques}

This section describes various models used for constructing predictions. Constructing prediction model requires the characteristics of program features. The program features may be static or dynamic. In these modelling techniques, we have considered dynamic program features for an application to be optimized. Dynamic program features were collected by compiling and executing benchmark applications using gcc or g++ compiler.

Constructing prediction model requires: 1 . Dynamic program feature extraction i.e performance counter values 2. Generating training data using random number generator strategy. 3. Append performance counter values and optimization sequence together and 4 . Use this model for an unseen program. For collecting performance counters HPCToolkit and PAPI interface ${ }^{13}$ were used. The performances counter values are collected by compiling each program with $-\mathrm{o} 0$ optimization level of GCC compiler. GCC compiler provides different levels of optimization techniques. Using random number generator strategy, 500 optimization sequences were generated from 65 optimization techniques. Using PAPI, the performance counter values collected for an application are: Branch related instructions: Level 1 cache memory related instructions and Level 2 cache memory related instructions; Floating point related instructions; Hardware interrupts and stalls instructions; Translation look aside buffer and Data Translation look aside buffer instructions.

\section{Total Instructions}

\subsection{Linear Modelling}

With this modelling technique we find a bit vector and construct a separate model for each and every optimization sequence. The input to this model is a set of performance counter values and the output is predicted by the probability in which particular optimization technique achieves optimization or not. The above steps are repeated for each and every optimization sequence. Models are created for all the optimization techniques. Best optimization techniques for a bench mark applications are taken related to best speed up. Once the model is trained, we can use this model to predict best sequence for an unseen program. For an unseen program the input to the model is performance counter values of a program and all 500 optimization sequences. The output is a probability $\mathrm{p}_{\mathrm{i}}$ for each optimization sequence whether it is turn ON or OFF. Thus, for all the models forms a probability function. Then it is sampled to generate optimization sequence to apply for an application. Figure 1 shows the functionality of linear modelling. 


\subsection{Iterative Modelling}

In this model the input is performance characterization of benchmark applications and various optimization sequences. Speed up is measured for each and every optimization sequence. The training data for this model is 500 random optimization sequences. All these optimization sequencess are applied to each and every benchmark applications. Speed up is measured for each and every optimization sequence with respect to default compiler settings. It is the ratio between the time required for base time and the time required to fine tune the code. The sequence which gives maximum speed up is noted for every benchmark applications. Once this model is trained, we can use this model to predict good optimization sequence for an unseen program based on maximum speed up.

\subsection{Proposed Modelling}

We have proposed multi variant modelling which is a combination of linear modelling and iterative modelling. The proposed modelling is explained in figure 2 . The input to the model is performance counter values of a benchmark program and three optimization sequences together are applied. The output is about which optimization sequences give better speed up than others. Measure the speed up for those three sequences with relative to default compiler settings. If speed up (s1) is greater than speed up (s2) and speed up (s3), then select sequence 1 . Else check the speed up (s2) greater than speed up (s3) then s2; else s3.

This model consists of four tuples. They are: PC, o1, 02 and o3, where, PC is the Performance Counter values;

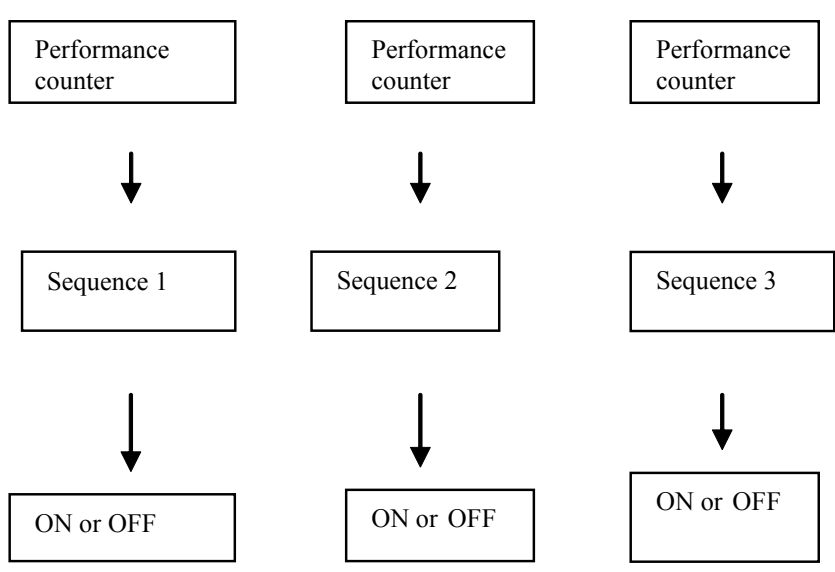

Figure 1. Linear modelling.
$\mathrm{O}_{1}$ is sequence $1 ; \mathrm{O}_{2}$ is sequence $2 ; \mathrm{O}_{3}$ is sequence 3 . Each time the better of three sequences are considered.

The comparison is performed in the following way: This model can predict good optimization sequences effectively and is trained using machine algorithm. Once trained, this model predicts good optimization sequence for an unknown program.

Based on the selection of best optimization sequences we can find optimal ordering effectively. Optimal ordering impacts program performance. Result shows that multi variant modelling predicts better sequence for both seen and unseen programs.

\subsection{Constructing Models}

Figure 3 shows the steps used for collecting performance counter values. Set of benchmark applications

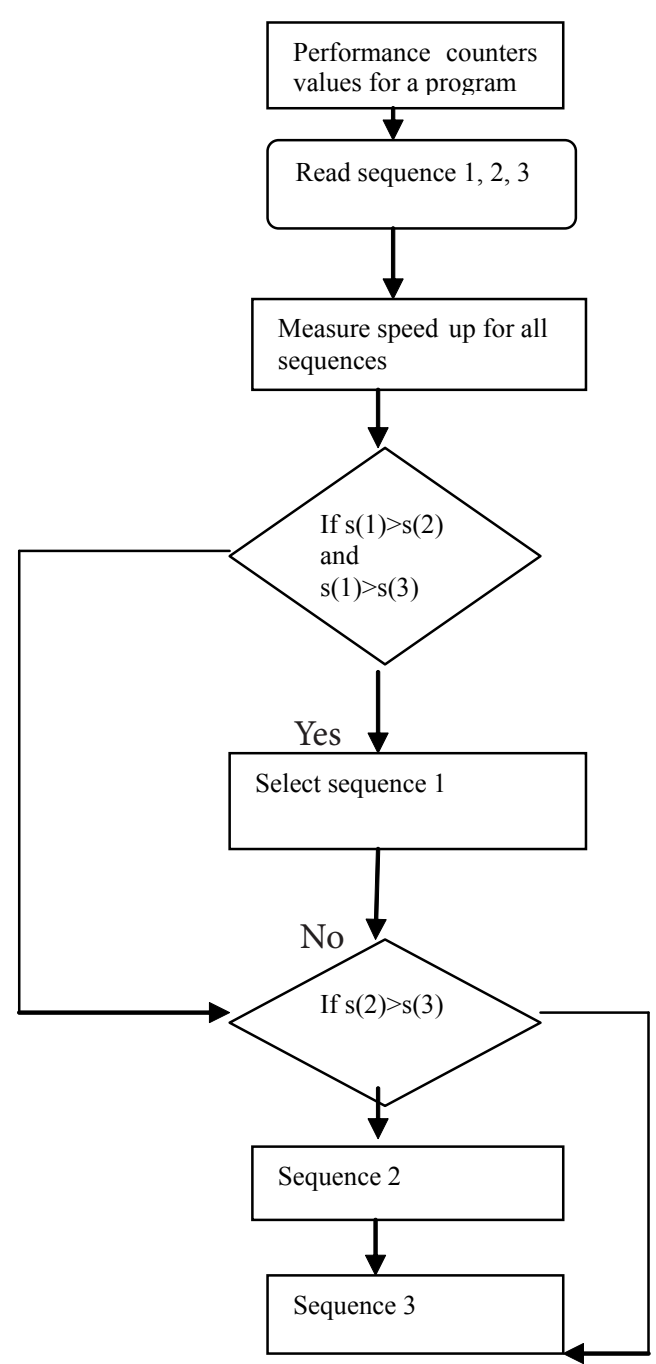

Figure 2. Overview of multi variant modelling. 


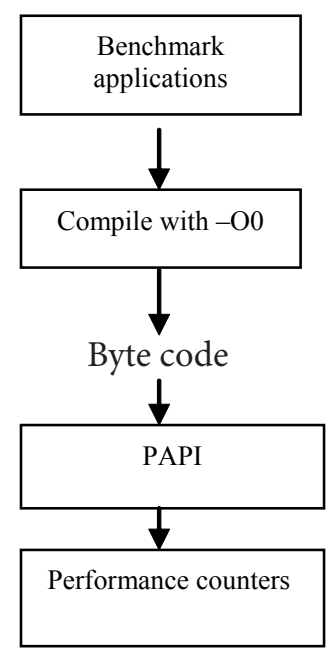

Figure 3. Collecting performance-counter values.

are compiled with gcc compiler without applying any optimizations. Using PAPI, interface performance counters are collected specific to architecture.

\subsection{Finding Speed Up}

Benchmark application is compiled with gcc compiler without applying any optimizations along with optimization sequence. Speed up is measured. The same procedure is repeated for all the sequences. Store all the optimization sequences along with its speed up in a database. Combine performance counters for a N-1 benchmark program and optimization sequences together along with its speed up given to a machine learning algorithm. Then the model learns itself. Once the method is learnt, the model predicts good optimization sequence for an unknown program.

\section{Machine Learning}

\subsection{Support Vector Machine Model}

It is a supervised learning algorithm. It can be used to classify the problem into linearly separable classes. SVM is used to transform the training data into various classes and then classifier is used to construct points in to a multiple classes.

\subsubsection{Regression Modelling}

Standard machine learning algorithm is used for predicting good optimization sequence for an unseen program.

\subsubsection{K-Nearest Neighbour}

It is a classification algorithm. Each test point is classified according to the $\mathrm{K}$ training points with feature most similar to the test point.

\subsubsection{Decision Trees}

It is a classification algorithm. The features of the program are considered as test point. Then decisions are taken, from root node to leaf node which yields classification for the test point.

\section{Results and Discussions}

We have conducted experiments using Intel Core2 Duo T6600 CPU 2.2Ghz. with 3GB DDR2RAM, L1cache $64 \mathrm{~KB}$, L2cache $2 \mathrm{MB}$, using ubuntu11.10 operating system, GCC compiler 4.5.2 $2^{12}$. Performance counters are collected using PAPI and HPC Toolkit. Multi variant modeling was constructed and trained using the above mentioned training algorithm. We used some of the Mibench ${ }^{9}$ Benchmark Application for conducting experiments. They are: basic math, bitcount, qsort, patricia, dijkstra and string search. Evaluated multi variant modeling is applied on both seen and unseen programs. To evaluate various modeling techniques, leave one out cross validation is used, i.e., $\mathrm{N}-1$ benchmark training points are tested on the $\mathrm{N}^{\text {th }}$ benchmark applications. Speed up is evaluated for all the modeling techniques. After measuring speed up for all the models, top 100 optimization sequences were selected which gave maximum speed up. Totally 500 random optimization sequences checked for every benchmark applications. It evaluates the results using all the machine learning algorithms. The results for all the modeling techniques using SVM, Regression Modeling-NN and Decision Trees are shown. The iterative modeling using regression works better when compared to multi variant modeling and linear modeling during first evaluation. When the evaluation increases from $5,10,50,100$, then multi variant modeling and iterative modeling performs well than linear modeling. Multi variant modeling achieves better performance when trained using support vector machine model. When the evaluation increases it achieves higher speed up than linear and iterative model. When compared to other training algorithms such as decision trees and $\mathrm{K}-\mathrm{NN}$, the model which uses SVM achieves higher performance. Regardless of learning algorithm, multi variant modeling achieves $78 \%$ performance over default settings. When 
the evaluation increases it achieves more than $90 \%$. The following figure (Figure 4) shows the maximum speed up obtained using top predicted sequences. Two different predictors are evaluated using SVM. One using set of trained optimization sequences where the application is not seen and the other using set of unseen sequences where the application is not seen.

Figure 4 shows multi variant modeling which outperforms most of the benchmark applications. The model was trained using support vector machine model for a set of trained optimization sequences. These trained optimization sequences are applied to a set of benchmark applications which is left out in Leave Out Cross Validation Model. It achieves nearly $12 \%$ performance improvement with respect to default compiler setting. Better performance is achieved even unseen sequences which also trained using SVM.

\section{Conclusion}

In this paper we have proposed multi variant modeling. This model is compared with other modeling techniques such as linear modeling and iterative modeling. All these models are evaluated using machine learning algorithm. Multi variant modeling can predict good optimization sequences using performance counter values. All the models are evaluated using mibench benchmark applications. The result shows that multi variant model and iterative model outperforms than model using linear model during initial evaluation. When the evaluation increases multi variant model outperforms linear and iterative modeling. We achieved $12 \%$ performance improvement for a trained sequence applied to an unseen benchmark application. In future multi variant model is extended by considering more benchmark applications.

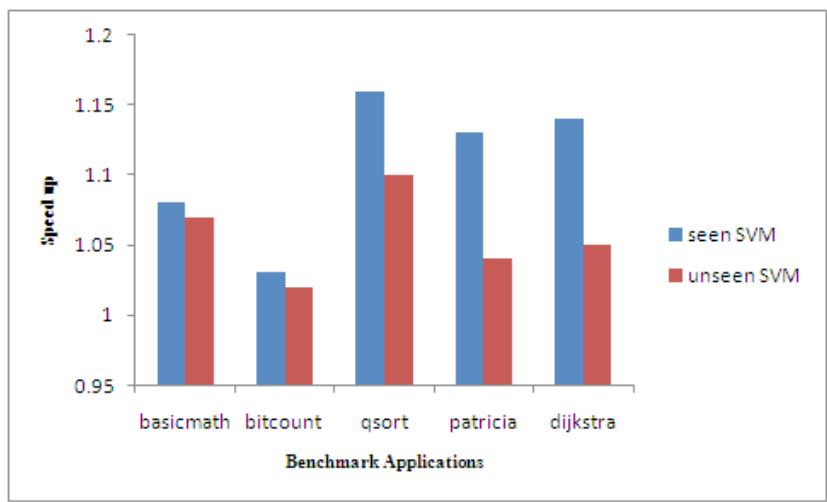

Figure 4. Speed up between seen SVM and unseen SVM.

\section{References}

1. Fursin G, Kashnikov Y, Memon AW, Chamski Z, Temam O, Namolaru M, Yom-Tov E, Mendelson B, Zaks A, Courtois E, Bodin F, Barnard P, Ashton E, Bonilla E, Thomson J, Williams CKI, O’Boyle M. Milepost GCC: machine learning enabled self tuning compiler. Int J Parallel Program. 2011; 39(3):296-327. doi:10.1007/s10766-010-0161-2.

2. Kulkarni PA, Whalley DB, Tyson GS. Evaluating heuristic optimisation phase order search algorithms. International Symposium on Code Generation and Optimization, CGO '07. 2007. p. 157-169. doi:10.1109/CGO.2007.9.

3. Agakov F, Bonilla E, Cavazos J, Franke B, Fursin G, O’Boyle MFP, Thomson J, Toussaint M, Williams CKI. Using machine learning to focus iterative optimization. International Symposium on Code Generation and Optimization, CGO '06. 2006. p. 295-305. doi:10.1109/CGO.2006.37

4. Cavazos J, O’Boyle M. Method specific dynamic compilation using logistic regression. Proceedings of the 21st annual ACM SIGPLAN Conference on Object-Oriented Programming Systems, Languages, and Applications, OOPSLA '06. 2006 Oct. p. 229-240.

5. Triantafyllis S, Vachharajani M, Vachharajani N, August DI. Compiler optimization-space exploration. International Symposium on Code Generation and Optimization, CGO '03. 2003 March 23-26. 2005. p. 204-215. doi:10.1109/CGO. 2003.1191546.

6. Almagor L, Cooper KD, Grosul A, Harvey TJ, Reeves SW, Subramanian D, Torczon L, Waterman T. Finding effective compilation sequences. Proceedings of the 2004 ACM SIGPLAN/SIGBED Conference on Languages, Compilers, and Tools for Embedded Systems, LCTES '04; New York (NY), USA: ACM; 2004. p. 231-239.

7. Kulkarni P, Hines S, Hiser J, Whalley D, Davidson J, Jones D. Fast searches for effective optimization phase sequences. Proceedings of the ACM SIGPLAN 2004 Conference on Programming Language, Design and Implementation, PLDI '04; New York (NY), USA: ACM; 2004. p. 171-182.

8. Stephenson M, Amarasinghe SP, Martin MC, O’Reilly UM. Meta optimization: improving compiler heuristics with machine learning. Proceedings of the ACM SIGPLAN 2003 Conference on Programming Language, Design and Implementation, PLDI '03; New York (NY), USA: ACM; 2003. p. 77-90.

9. Guthaus MR, Ringenberg JS, Ernst D, Austin TM, Mudge T, Brown RB. Mibench: a free commercially representative embedded benchmark suite. IEEE $4^{\text {th }}$ Annual Workshop on Workload Characterization (WWC-4); Washington DC, USA: IEEE Computer Society; 2001 Jun. p. 3-14. doi:10.1109/WWC.

10. Cooper KD, Schielke PJ, Subramanian D. Optimizing for reduced code space using genetic algorithms. Proceedings 
of the ACM SIGPLAN 1999 Workshop on Languages, Compilers, and Tools for Embedded Systems, LCTES '99; New York (NY), USA: ACM; 1999. p. 1-9.

11. Ng AY. On feature selection: learning with exponentially many irrelevant features as Training examples. Proceedings of the Fifteenth International Conference on Machine Learning. 1998. p. 404-412. doi:10.1.1.48..5103.

12. GCC online documentation. Available from: http://gcc. gnu.org/onlinedocs/
13. PAPI: A Portable Interface to Hardware Performance Counters. http://icl.cs.utk.edu/papi

14. Andrews J, Sasikala T. Evaluation of various compiler optimization techniques related to Mibench benchmark applications. J Comput Sci. 2013; 9(6):749-756.

15. Andrews J, Sasikala T. A novel approach for selection of best set of optimization functions for a benchmark applications using an effective strategy. Indian Journal of Computer Science Engineering. 2013; 4(1):65-71. 